\title{
Fatores que influenciam o tempo de gestação em éguas - Revisão de literatura
}

\author{
Factors affecting gestation length in mares - Literature review \\ Factores que influyen en el tempo de gestación em yeguas - Revisión de literatura
}

\section{Resumo}

O tempo de gestação (TG) na espécie equina é uma variável fisiológica de grande importância econômica e clínica uma vez que o manejo desses animais nos criatórios demanda um conhecimento preciso quanto ao momento do parto. Esta variável apresenta uma ampla variação em relação as outras espécies de produção, sendo considerado fisiológico o intervalo entre 320 e 390 dias de período gestacional na égua. Descreve-se que o tempo de gestação pode ser influenciado por diversos fatores maternos (idade materna, número de partos, nutrição materna), fetal (sexo do potro) e ambientais (local, ano do parto, mês do parto, clima. Dessa forma, este estudo tem o objetivo de apresentar uma revisão dos fatores que podem influenciar o tempo de gestação na espécie equina, sendo estes os fatores maternos, fetais, paternos e ambientais. Conclui-se os fatores maternos e fetal já são bem elucidados quanto a sua relação com o tempo de gestação, porém a influência do garanhão, os fatores ambientais e climáticos ainda necessitam de mais estudos na espécie equina.

Palavras-chave: Obstetrícia; Potros; Paridade; Fatores climáticos.

\begin{abstract}
Gestation length (TG) in horses is a physiological variable of great economic and clinical importance. For the management in breeding farms requires precise knowledge as to the time of parturition. This variable has a wide variation in comparison with other production animals, it has been considered physiological the interval between 320 to 390 days of gestational. Gestation length can be influenced by several maternal factors (maternal age, number of births, maternal nutrition), fetal (sex of the foal) and environmental factors (location, year of birth, month of birth, weather. Thus, this study aims to present a review of the factors that can influence the length of pregnancy, these being the maternal, fetal, paternal and environmental factors. It is concluded that maternal and fetal factors are already well elucidated as to their relationship with the time of pregnancy, however influence of the stallion, environmental and climatic factors still need further studies in equine specie.
\end{abstract}

Keywords: Obstetrics; Foals; Parity; Climatic factors.

\section{Resumen}

El tiempo de gestación (TG) en la especie equina es una variable fisiológica de gran importancia económica y clínica ya que el manejo de estos animales en granjas exige un conocimiento preciso del momento del parto. Esta variable presenta una amplia variación en relación a las otras especies de producción, considerándose fisiológico un intervalo entre 320 y 390 días de gestación en la yegua. Se describe que el momento del embarazo puede estar influenciado por varios factores 
maternos (edad materna, número de partos, nutrición materna), fetales (sexo del potro) y factores ambientales (ubicación, año de nacimiento, mes de nacimiento e clima). Así, este estudio tiene como objetivo presentar una revisión de los factores que pueden influir en el tiempo de gestación en la especie equina, siendo estos los maternos, fetales, paternos, y ambientales. Concluye que los factores maternos y fetales ya están bien esclarecidos en cuanto a su relación con el tiempo de gestación, sin embargo la influencia del semental, los factores ambientales y climáticos aún necesitan más estudios en la especie equina.

Palabras clave: Obstetricia; Potros; Paridad; Factores climáticos.

\section{Introdução}

O tempo de gestação (TG) é uma variável fisiológica de importância econômica e clínica na espécie equina, uma vez que o manejo de animais de alto valor demanda um conhecimento preciso quanto ao momento do parto (Silver, 1990).

Alguns fatores podem influenciar o tempo gestacional em éguas, são descritos os fatores maternos, fetais e ambientais (DaviesMorel et al., 2002; Satué et al., 2011). Dentre os fatores maternos, incluem-se a idade da égua (Wilsher \& Allen, 2003; Valera et al., 2006; Langlois \& Blouin, 2012) e o número de partos (Pool-Anderson et al., 1994; Panchal et al., 1995). O fator fetal gênero do potro também influência o tempo de gestação (Hevia et al., 1994; Panchal et al., 1995). Os fatores ambientais que influenciam o tempo de gestação são o mês de concepção ou do parto (Sanchez, 1998; Ousey et al., 2000; Pérez et al., 2003; Sevinga et al., 2004), o clima, assim como o ano do parto (Pérez et al., 2003; Cilek, 2009).

Considerando o nascimento de um potro viável, o TG na espécie equina varia de 320 - 390 dias de gestação, sendo essa grande variação devido a raça e a população estudada (Silver, 1990; Panchal et al., 1995, Immergart, 1997). O conhecimento do TG é clinicamente importante, pois assim como em humanos, é utilizado para avaliar a viabilidade fetal após o parto, por auxiliar na definição de prematuridade ou dismaturidade nos potros neonatos (Koterba et al., 1990; Pieszak et al., 2020).

Dessa forma, este estudo tem o objetivo de apresentar uma revisão dos fatores que podem influenciar o tempo de gestação sendo estes os fatores maternos, fetais, paternos e ambientais.

\section{Metodologia}

O presente estudo consta de uma revisão de literatura qualitativa como ferramenta de metodologia de pesquisa científica (Pereira et al., 2018), realizando assim uma reflexão sobre a manifestação de ideias e sua representação sobre os fatores que influenciam o período de gestação em éguas. Para realização da revisão foram selecionados artigos utilizando as plataformas Mendeley, MEDLINE, PubMed e SciELO. Devido ao escasso número de publicações específicas sobre o tema na espécie equina, foram utilizados artigos publicados a partir do ano de 1961.

\section{Fatores Maternos}

Dentre os fatores maternos conhecidos por influenciar o tempo de gestação em éguas, destacam-se o estado nutricional, a idade materna e a sua paridade, sendo essa definida pelo número de partos que a progenitora teve em sua vida reprodutiva (Davies Morel et al., 2002; Satué et al., 2004; Winter et al., 2007). Ainda, o fator genético individual é influente na duração da gestação, sendo descrito um índice de 25,8\% da variabilidade do TG relacionado a própria égua (Satué et al., 2011).

A idade materna foi considerada um fator importante para o tempo de gestação por diversos autores (Satué et al., 2004; Valera et al., 2006; Aoki et al., 2013, Ferreira et al., 2016). Na sua maioria demonstram que quanto maior a idade materna, maior o tempo de gestação (Platt, 1979; Demirci, 1988; Langlois \& Blouin, 2012). Contudo, Valera et al. (2006) observaram que a duração da gestação diminui à medida que a égua envelhece, com os menores períodos de gestação ocorrendo quando a égua tem 10-12 anos, a partir dos 13 anos de idade o tempo de gestação apresenta um aumento gradativo. 
$\mathrm{Na}$ espécie equina o envelhecimento materno pode estar relacionado com a redução da capacidade de interação fetoplacentária, devido a diminuição da nutrição uterina (Rossdale et al., 1982; Wilsher \& Allen, 2003) ou deficiência do metabolismo hormonal para o crescimento fetal (Gluckman \& Hanson, 2004), alterando assim o TG. A paridade, também é descrita por diversos autores por influenciar o TG (Poll-Anderson et al., 1994; Cacic et al., 2002). Pool-Anderson et al. (1994), descrevem que éguas primíparas apresentam maior período gestacional comparadas as multíparas. Em outro estudo Ferreira et al., (2016), demonstram que a paridade materna afetou o período gestacional para éguas primíparas até a terceira gestação. Sendo as gestações mais curtas entre a $4^{\mathrm{a}}$ e a $8^{\mathrm{a}}$ parição.

Correlacionando a idade e paridade materna, Wilsher e Allen (2003) demonstraram que estes dois fatores influenciam o desenvolvimento e densidade dos microcotilédones, sendo ambos menores em éguas idosas e multíparas. Sendo assim, podem estar envolvidos no fato dessa categoria de éguas apresentar maior tempo de gestação para garantir o completo desenvolvimento do potro ao nascimento.

\section{Fator Fetal}

Diversos autores já descreveram o gênero do potro como sendo o fator fetal mais importante, sendo observada gestações de 1-2 dias mais longas em éguas prenhes de potros do sexo masculino, incluindo diversas raças como Andaluz, Frísio, Purosangue Inglês, raças de tração pesada e Mangalarga paulista (Pérez et al., 2003; Sevinga, et al., 2004; Van Rijssen et al., 2010; Aoki et al., 2013; Ferreira et al., 2016). Valera et al., (2006) mostraram que o gênero influencia 0,43\% do total de variação no TG de éguas da raça Andaluz. Em contrapartida, poucos estudos como El-Wishy et al., (1990) não encontraram esta diferença no tempo de gestação relacionada ao sexo.

A razão para a variação no TG associada ao gênero do potro ainda não foi completamente elucidada, a primeira hipótese descreve que a produção de testosterona pelo feto macho seja superior em relação a fêmea, assim como os machos apresentem um maior desenvolvimento corporal (Cilek, 2009). Existe ainda a hipótese de que a diferença em dias de gestação de acordo com o sexo do potro poderia expressar as diferenças no controle endócrino do parto, portanto, como o parto ocorre quando o desenvolvimento fetal está completo, o TG de potros machos seria mais longo por tratar-se, geralmente, de indivíduos maiores ao nascimento (Wilsher \& Allen, 2003).

\section{Fatores Ambientais}

Os fatores ambientais conhecidos por alterar o TG são: mês de concepção/ parto (Ousey et al., 2000; Pérez et al., 2003; Sevinga et al., 2004; Cilek, 2009; Satué et al., 2011; Langlois e Blouin, 2012), o ano do parto (Pérez et al., 2003; Valera et al., 2006; Cilek, 2009) e local do parto (Valera et al., 2006; Cilek, 2009).

\subsection{Mês e Ano do parto}

O mês da concepção, na maioria dos estudos, apresenta influência no TG. Sendo observada uma redução do período gestacional nas éguas acasaladas ao final da estação reprodutiva em comparação com as éguas acasaladas no início da temporada (Sánchez, 1998; Satué, 2004; Cilek, 2009), em especial no hemisfério norte (Pérez et al., 2003).

Valera et al. (2006) apresentaram que este fator é responsável por 4,4\% da variação no TG de reprodutoras das raças Andaluz e Árabe no sul de Espanha. A relação do mês da concepção/parto pode ter relação ao fato da sazonalidade do equino e fatores climáticos que alterariam as condições de alimentação. Desta forma propõem a proximidade da primavera como momento ideal para a ocorrência do parto, promovendo assim gestações mais longas ou mais curtas como forma de aproximar que o parto ocorra na primavera (Evans \& Torbeck, 1998). O que está de acordo com os autores que descrevem a variação no TG decorrendo 
da melhor nutrição, condições de temperatura e influência da luz solar no mês do parto, característicos da primavera (Campitelli et al., 1982; Macpherson \& Paccamonti, 2011).

O fator ano do parto também foi observado por influenciar o TG na espécie equina (Pérez et al., 2003; Cilek, 2009; Langlois \& Blouin, 2012). Autores acreditam que o ano tem relação com TG por refletir a variação que pode ocorrer na condição nutricional anualmente, especialmente em animais provenientes de criações extensivas (Davies Morel et al., 2002; Pérez et al., 2003; Valera et al., 2006; Langlois \& Blouin, 2012). Assim, o manejo nutricional dos animais pode reduzir esse impacto, como o descrito por Satué et al. (2011), os quais não encontraram influência do ano em relação ao TG, devido a população do estudo ter a oferta de alimentação balanceada e assim não sofrer influência severa das variações nutricionais das pastagens.

Outra hipótese está relacionada as variações climáticas que podem ocorrer, sendo que anos secos e/ou com temperaturas elevadas levariam a tempos de gestação mais longos (Valera et al., 2006). Porém outros autores relatam tempo de gestação maiores em anos com temperaturas mais baixas (Pérez et al., 2003; Cilek, 2009).

\subsection{Fatores climáticos}

Fatores climáticos: temperatura, umidade relativa, fotoperíodo e a velocidade do vento são variáveis climáticas que podem alterar a fisiologia de grandes animais (West, 2003). Em equinos a temperatura, umidade e as horas de luz diária são consideradas como possíveis influências no tempo de gestação (Davies Morel et al., 2002)

Ambientes com temperatura e umidade elevadas são descritas por influenciar de forma negativa os índices reprodutivos em animais de produção em países tropicais e subtropicais (Ju, 2005). Aoki et al. (2013) analisaram as temperaturas mínimas e máximas nos últimos 10 dias gestacionais em éguas de raças de tração pesada, porém não encontraram relação com o TG.

Corvalan et al. (1961), em seu estudo com éguas Puro-Sangue Inglês no Chile, identificaram que a temperatura média de 13,5 ${ }^{\circ} \mathrm{C}$, pode ser considerada um ponto de corte quando avaliada sua relação com o TG na espécie equina. Sendo que temperaturas abaixo de $13,5{ }^{\circ} \mathrm{C}$ apresentaram correlação positiva com o TG, enquanto valores superiores a esse demonstraram uma correlação negativa com a duração da gestação. Ainda no mesmo estudo a variação isolada de umidade não demostrou uma relação com o tempo de gestação.

Quando avaliada a eficiência reprodutiva na espécie equina, foi demonstrado que a zona de conforto térmico para a espécie é de $5^{\circ} \mathrm{C}$ a $25^{\circ} \mathrm{C}$ (Morgan, 1996). Jacob et al. (2019) em estudo no Brasil, verificaram que temperaturas acima de $24^{\circ} \mathrm{C}$ já promoveram o estresse térmico em equinos, ocasionado um efeito negativo sobre os índices reprodutivos, em especial as taxas de concepção.

Em éguas Puro-sangue Inglês, estudos preliminares sugerem uma correlação negativa dos valores de índice temperatura umidade (THI) no mês de parto com o TG em éguas Puro Sangue Inglês no hemisfério sul, indicando que estes fatores climáticos afetam o período gestacional (Curcio et al., 2017; Scalco et, al., 2018).

\subsubsection{Fotoperíodo}

O fotoperíodo, que pode ser definido como horas de iluminação diária, é um fator climático já estabelecido no controle da ciclicidade reprodutiva, devido a característica poliestrica estacional da espécie equina (Mckinnon et al., 2011). O despertar do ritmo circadiano endógeno na égua está associado a melatonina, uma vez que, essa diminui a concentração sérica em decorrência do maior período de luminosidade traduzindo assim a informação do fotoperíodo para a égua (Sharp, 1988). A redução da melatonina estimula a liberação de GnRH (hormônio liberador de gonadotrofinas) o qual promove a liberação sequencial das gonadotrofinas FSH e LH (FSH - hormônio folículo estimulante; LH - hormônio luteinizante), esse processo culmina com a ocorrência do período de estro e ovulação. Nas éguas não gestantes, esse processo se repete a cada 21 dias caracterizando a ciclicidade da égua, organizada pelo ritmo sazonal do fotoperíodo (Palmer e Guillaume, 1992). 
Em equinos, sugere-se que o aumento de horas de luz diária reduziria o TG (Hintz et al., 1992; Valera et al., 2006). Na mesma linha Hodge et al. (1982) demonstraram que éguas gestantes no hemisfério norte submetidas a 16 horas de luz diárias, no início de dezembro, adiantaram a data de parto. O mecanismo proposto para a ação da melatonina seria que o aumento do fotoperíodo sinalizaria a época do ano para o feto, o que poderia ocorrer via direta pelo envio de melatonina para a unidade fetoplacentária e ligação aos receptores de melatonina fetal expressas no cérebro e retina, ou indiretamente via alteração dos perfis endócrinos na égua (Reppert et al., 1985). Ainda é proposto que variações na luminosidade diária possam causar modificações marcadas na taxa de maturação fetal (Sharp, 1988), contudo ainda não existe comprovação dessa hipótese.

A utilização das máscaras de luz azul (Nolan et al., 2017) como ferramenta de aumentar o fotoperíodo nas éguas gestantes promoveu redução do TG e incrementou o processo de adaptação inicial do potro neonato. $\mathrm{O}$ único estudo até o presente momento no hemisfério sul com a utilização de iluminação artificial convencional, apresentou resultado diferente. Foi observado que o aumento no fotoperíodo artificial no mês pré-parto ( 15 horas de luz e 9 horas de escuridão por dia) não apresentou influência no TG, promovendo sim a redução do intervalo entre o parto e a primeira ovulação (Malschitzky et al., 2001).

\section{Local do Parto}

A área geográfica pode explicar as diferenças observadas em TG nos diferentes locais (Davies Morel et al., 2002; Winter et al., 2007). Já se observou em estudos que, raças equinas que se desenvolvem sua atividade reprodutiva em regiões semelhantes, tem TG semelhantes e são afetados por fatores semelhantes (Kurtz Filho et al., 1997; Davies Morel et al., 2002; Winter et al., 2007). O local é um fator ambiental pouco descrito por sua influência no TG em equinos, sendo muitas vezes direcionado as diferenças climáticas de cada localidade, contudo autores descrevem as variações do TG influenciada pela diferença de latitude e pressão atmosférica dos locais em que as éguas desenvolveram as gestações independente das diferenças climáticas individuais (Kurtz Filho et al.,1997; Davies Morel et al., 2002; Winter et al., 2007).

\section{Fatores Paternos}

O valor de herdabilidade do garanhão em relação ao tempo de gestação foi estimado entre $0,19 \%$ e 0,38 \% (Valera et al., 2006; Satué et al., 2011; Langlois \& Blouin, 2012).

A maior parte dos autores não encontraram uma influência paterna afetando o TG em equinos (Morel et al., 2002; ElWishy et al., 1998; Ferreira et al., 2016). Em contraste, Marteniuk et al., (1998) demostraram que certos garanhões promoveram gestações menores que 340 dias, enquanto o TG com outros foi associado a gestações com duração maior que 350 dias. Ainda associada a esta relação positiva entre pai e duração gestacional, Satué et al. (2011) mostraram que alguns garanhões tendem a produzir gestações que são menores ou maiores do que 340 dias, sugerindo que garanhões que tendem a prolongar a duração da gestação deve ser usada no início de a época de reprodução.

Contudo a relação específica entre o garanhão utilizado no cruzamento e o TG, de forma objetiva em relação a idade ou linhagem específica nas raças ainda não está completamente elucidada na literatura.

\section{Conclusão}

As informações da literatura revisada demostraram que o tempo de gestação na égua está sujeito a uma grande variação, devido ao efeito de múltiplos fatores. Considerando os fatores maternos, a idade e número de partos da égua são os mais relevantes, éguas multíparas com mais de 14 anos apresentam as gestações mais longa. O sexo do potro é o fator fetal principal, sendo que gestações de potros machos são mais longas do que as gestações de potros fêmeas. Em relação aos fatores paternos e 
ambientais os resultados são ainda contraditórios e necessitam de mais aprofundamento para definição de como eles influenciam o tempo de gestação na espécie equina.

\section{Referências}

Aoki, T., Yamakawa, K., \& Ishii, M. (2013). Factors affecting gestation length in heavy draft mares. J. Equine Vet. Sci. 33, 437-440.

Cacic, M. P., Caput, P., \& Ivamkovic, A. (2002). Influence of environmental and genetic factors on the reproductive characteristics and fertility of Posavina mares. Stocarstvo. 56(4), 243-256.

Campitelli, S., Carenzi, C., \& Verga, M. (1982). Factors which influence parturition in the mare and development of the foal. Appl Anim Ethol, 9: 7-14.

Cilek, S. (2009) The survey of reproductive success in Arabian horse breeding from 1976 to 2007 at Anadolu State Farm in Turkey. J Anim Vet Adv. 8, 389-396.

Curcio, B. R., Moraes, B. S. S., Canisso, I. F., Lima, F. S., Silva, G. C., \& Nogueira, C. E. W. (2017). Duration of gestation in Thoroughbred mares kept under tropical and subtropical climate. Clinical Theriogenology; v.3, 450.

Corvalan, R. A., \& Girardi, E. H. (1961). Influencia de algunos factores climáticos sobre la duración de la gestación de yeguasfina sangre de carrera. Zooiatria ;2, $37-58$.

Davies Morel, M. C. G., Newcombe, J. R., Holland, S. J. (2002). Factors affecting gestation length in the Thoroughbred mare. Anim Reprod Sci. v.74, 175-185.

Demirci, E. (1988) Length of gestation period in purebred Arab mares and correlation between age and gestation length. A.U. Vet. Fank. Derg. v.35, 69-79.

El-Wishy, A. B., El-Sayed, M. A. I, Seida, A. A., Ghoneim, I. M., \& Serur, B. H. (1990). Some aspects of reproductive performance in Arabian mares in Egypt. Reprod Domest Anim. 25, 227-234.

Evans, W. J., \& Torbeck, R. L. (1998). Breeding management and foal development: Equine Res. Incorp. Texas. p. 700.

Ferreira, J. R. M., Meirelles, M. G., Guimarães, C. F., Alonso, M. A., Nichi, M., \& Fernandes, C. B. (2016). Factors affecting gestational length in the Mangalarga Paulista breed. Anim Reprod. v.13, 117-121.

Gluckman, P. D., \&Hanson, M. A. (2004). Restrição materna do crescimento fetal e suas consequências. Seminários em Medicina Fetal e Neonatal, 9(5), 419-425.

Hevia, M. L., Quiles, A. J., Fuentes, F., \& Gonzalo, C. (1994). Reproductive performance of Thoroughbred mares in Spain. J. Equine Vet Sci. 14(2), 89-92.

Hintz, H. F., Williams, J., Hollman, R., Housay, H., \& Ferrer, R. (1992). Effect of month of breeding on duration of gestation period of Thoroughbred. Equine Pract. 16-20.

Hodge, S. L., Kreider, J. L., Potter, G. D., Harms, P. G., \& Fleeger, J. L. (1982). Influence of photoperiod on the pregnant and postpartum mare. Am J Vet Res. v.43, 1752- 1755

Immegart, H. M. (1997). Abnormalities of pregnancy In: Yougquist, R.S. (Ed.), Current Therapy in Large Animal Theriogenology, Saunders, Philadelphia, USA $113 \mathrm{p}$.

Jacob, J. C. F., Oliveira, J. P., Ferraz, P. J., \& Guerson, Y. B. (2019). Estresse térmico na reprodução equina. Rev Bras Reprod Anim.43(2), $216-221$.

Ju, J. C. (2005.) Cellular responses of oocytes and embryos under heat stress: hints to molecular signaling. Anim Reprod. 2(2), 79-90.

Koterba, A. M., Drummond, W. H., \& Kosch, P. C. (1990). Equine Clinical Neonatology. Lea \& Febiger, Philadelphia, London.

Kurtz Filho, M., Deprá, N. M., Alda, J. L., Castro, I. N., Corte, F. D., \& Silva, C. A. M. (1997). Duração da gestação em relação a idade de Éguas de raça Puro Sangue de Corrida, aos pesos do potro e da placenta, e ao horário do parto. Braz. J. Vet. Anim. Sci. 34(1), 37-40.

Langlois, B., Blouin, C. (2012). Genetic parameters for gestation length in French horse breeds. Livestock Sci. 142, $133-139$.

Macpherson, M. L, \& Paccamonti, D. L. (2011). Induction of parturition. In: McKinnon AO, Squires EL, Vaala WE, Varner DD (Ed.). Equine Reproduction. 2nd. Oxford, UK: Wiley-Blackwell, 2262-2267.

Malschitzky, E., Schilela, A., Meirelles, L. S., Mattos, A. L. G., Gregory, R. M., \& Mattos R. C. (2001). Artificial photoperiod in pregnant mares and its effect on pregnancy length and postpartum reproductive performance. Pferdeheilkunde. v.17, 565-69.

Marteniuk, J. V., Carleton, C. L., Lloyd, J. W., \&Shea, M. E. (1998). Association of sex of fetus, sire, month of conception, or year of foaling with duration of gestation in Standardbred mares. J Am Vet Med Assoc. 212, 1743-45.

McKinnon, A. O., Squires, E. L., Vaala, W. E., \& Varner, D. D. (2011). Equine Reproduction. 2nd ed. Oxford: Wiley-Blackwell.

Morel, M. C. G. D., Newcombe, J. R., \& Holland, S. J. (2002). Factors affecting gestation length in the Thoroughbred mare. Anim Repr Sci. 74, $175-85$.

Morgan, K. (1996). Short-term Thermoregulatory Responses of Horses to Brief Changes in Ambient Temperature. Dissertation. Report 209. Swedish University of Agricultural Sciences Uppsala, Sweden, 51p. 
Nolan, M. B., Walsh, C. M., Duff, N., McCrarren, C., Prendergast, R. L., \& Murphy, B. A. (2017). Artificially extended photoperiod administered to pre-partum mares via blue light to a single eye: observations on gestation length, foal birth weight and foal hair coat at birth. Theriogenology. 100, 126-33.

Ousey, J. C., Rossdale, P. D., Palmer, L., Grainger, L., \& Houghton, E. (2000). Effects of maternally administered depot ACTH 1-24 on fetal maturation and the timing of parturition in the mare. Equine Vet J. 32(6), 489-496.

Palmer, E., \& Guillaume, D. (1992). Photoperiodism in the equine species-what is a long night? Anim Reprod Sci. 28, 21-30.

Panchal, M. T., Gujarati, M. L., \& Kavani, F. S. (1995). Some of the reproductive traits in Kathi mares in Gujarat State. Indian J Anim Reprod. v.16.

Pereira, A. S., Shitsuka, D. M., Parrreira, F. J., \& Shitsuka, R. (2018). Metodologia da pesquisa científica. $1^{\circ}$ Edição UBA/NTE/UFSM, Universidade de Santa Maria.

Pérez, C. C., Rodríguez, I., Mota, J., Dorado, J., Hidalgo, M., Felipe, M., \& Sanz, J. (2003). Gestation length in Carthusian Spanishbred mares. Livest Prod Sci. 82, $181-187$

Pérez, C. C., Rodríguez, I., Sanz, J., Acosta, M., \& Valera, M. (1997). Factores que influyenen la duración de la gestación y el peso de la placenta al parto em el P.R.E. de Estirpe Cartujana. Arch Reprod Anim. 3, 78-85.

Pieszak, G. M., Gomes, G. C., \& Rodrigues, A. P. (2020). Fatores que interferem no processo de parto e nascimento: revisão interativa da literature. Research, Society and Development, $9(7)$.

Platt, H. (1979). Growth of the equine foetus. Equine Vet. J. 16, 247-252.

Pool-Anderson, K., Raub, R. H., \& Warren, J. A. (1994). Maternal influences on growth and development of full-sibling foals. J. Anim. Sci. 72(7).

Reppert, S. M., Duncan, M. J., \& Duncan, B. D. (1985). Photic influences on the developing mammal. In: Evered D, Clork S, editors. CIBA foundation Symposium photoperiodism, melatonin and pineal. London: Pitman Publishing; 116-28.

Rossdale, P. D., \& Silver, M. (1982). The concept of readiness for birth. J ReprodFertil Suppl. 32, 507-510.

Sánchez, A. (1998). Contribución al studio de laduración de la gestación y surel acióncon el rendimento em el producto equino Fina Sangre de Carrera. Tesis Magíster en Ciencias. Universidad Austral de Chile.

Sánchez, A., Díaz, O., \& Gatica, R. (1999). Algunas consideraciones sobre laduración de lagestación em la yegua. Arch Reprod Anim. V.8, 18-23.

Satué, K. (2004). Hematología de layeguaP.R.E. de Estirpe Cartujana. Doctoral Thesis. CEU-Cardenal Herrera University. Department of Animal Medicine and Surgery. Faculty of Veterinary Medicine. Moncada. Valencia. Spain.

Satué, K., Felipe, M., Mota, J., \& Muños, A. (2011). Factors influencing gestational length in mares: A review. Livestock Sciense. 136(28), 287-294.

Scalco, R., Canisso, I. F., Silva, G. C., Almeida, T. L., Pazinato, F. M., Borba, L. A., Feijó, L. S., Nogueira, C. E. W., \& Curcio, B. R. (2018). Temperaturehumidity index (THI) is associated with gestation length in Trouroughbred mares. J Equine Vet Sci. v66, 254.

Sevinga, M., Barkema, H. W., Stryhn, H., \& Hesselink, J. W. (2004). Retained placenta in Friesian mares: incidence, and potential risk factors with special emphasis on gestational length. Theriogenology 61(5), 851-859.

Sharp, D. C. (1988). Transition into the breeding season: clues to the mechanism of seasonality. Equine Vet J. 20, $159-161$.

Silver, M. (1990). Prenatal maturation, the timing of birth and how it may be regulated in domestic animals. Exp Physiol. 75(3), 285-307, 1990.

Valera, M., Blesa, F., Santos, R. D., \& Molina, A. (2006). Genetic study of gestation in Andalusian and Arabian mares. Animal Reprod. Sci. 95, 75-96.

Van Rijsseni, J., Gee, E. K., Rogers, W., \& O’Brien, R. (2010). Factors influencing gestation length and interval from foaling to conception in mares managed on a commercial Thoroughbred studfarm. Proc. New Zealand Soc. Anim. 70, 146- 148.

West, J. W. (2003). Effects of heat-stress on production in dairy cattle. J. Dairy Sci. 2131-2144.

Wilsher, S., \& Allen, W. R. (2003). The effects of maternal age and parity on placental and fetal development in the mare. Equine Vet J. 35(5), 476-483.

Winter, G. H. Z., Rubin, M. I. B., Corte, F. D., \& Silva, C. A. M. (2007). Gestational length and first postpartum ovulation of Criollo mares on a stud farm in Southern Brazil. Journal of Equine Veterinary Science, v.27, 531-534. 